\title{
Literacy and Language
}

\section{Disentangling Measures of Access, Utilization, and Quality}

A 47-year-old man was found to have advanced lung cancer. After discussing options with his primary care physician, the patient decided in favor of palliative care. His physician referred him for home hospice. Two months later, the patient's brother called because the patient had been having intractable pain. Because the patient had lost his insurance, home hospice had rejected the referral and sent him to a social worker, who gave the patient a Medicaid application. Neither the patient nor his brother had been able to read or complete the form. The brother eventually submitted the form with the assistance of the physician. Two weeks after the patient died, his brother received a letter informing him that the patient had qualified for Medicaid.

A Spanish-speaking woman with claudication was found by her primary care physician to have a $5.5 \mathrm{~cm}$ abdominal aortic aneurysm (AAA) and was referred for consideration of surgery. A consultation was faxed to the Surgery Department and a copy given to the patient. At the follow-up visit, when the physician asked her whether she had decided to proceed with surgery, the patient informed her physician that no surgery was necessary. The surgeon's note in the chart described the claudication symptoms and recommended the patient increase her walking; there was no mention of the AAA. The patient acknowledged that she had not handed the consultation form to the surgeon because she couldn't read it and did not know what it was for. The patient was re-evaluated, but the AAA had expanded to the renal arteries. The patient ultimately went to surgery but did not survive the operation.

An elderly Cantonese-speaking woman was hospitalized because she had taken too much warfarin. Late that night, she got up to go to the bathroom. A nurse on duty stopped her and tried to get her back into bed. When the patient persisted in wanting to go to the bathroom, the nurse thought she was agitated. Instead of getting an interpreter, the nurse had the patient put in restraints, and gave her a sedative. When she didn't respond immediately to the sedative, the staff gave her more. By morning, she was barely responsive.

A significant proportion of patients in the U.S., like those described above, have limited health literacy (HL), limited English proficiency (LEP), or both. The health care system is poorly designed to address these barriers, placing great literacy and language fluency demands on patients and their families. While a growing number of studies examine the effects of communication barriers on health care, many questions remain unanswered. Understanding the impact of literacy and language barriers on access, cost, and quality across health services can inform efforts to reduce health care disparities.
In this issue of the Journal of General Internal Medicine, Baker et al. linked claims data with literacy data collected as part of their landmark Prudential Health Literacy Study to determine whether limited HL impedes access to care among patients enrolled in a major Medicare managed care plan. ${ }^{1}$ Since limited HL has been shown to be associated with worse health status, examining the degree to which this relationship can be explained by access problems is critical. While qualitative studies have determined that patients with limited literacy commonly experience access barriers, such as difficulties completing paperwork and navigation problems, ${ }^{2}$ delays in seeking care, ${ }^{3}$ and Medicaid enrollment denials due to procedural reasons, ${ }^{4,5}$ the relationship between HL and appropriate use of health services remains largely unknown. ${ }^{6}$ Baker et al. found no independent association between HL and indicators of primary care access, such as time to first primary care physician visit, proportion without any visit, and mean number of annual primary care visits. While these results provide reassurance regarding the degree to which the door to the "primary care home" is open for Medicare managed care patients with limited HL, the conclusion that limited HL is probably not a major barrier to health care access should be interpreted with caution. Primary care utilization is most frequently used as an indicator of differences in access between insured and uninsured; its utility for measuring differences in access among a cohort of managed care enrollees may be more limited. As the authors point out, findings from this and other published Prudential HL studies that studied the same population corroborate this concern. Specifically, despite similar rates of primary care utilization, patients with limited HL were less likely to receive preventive services, ${ }^{7}$ more likely to use the emergency department, and more likely to require hospitalization. ${ }^{8}$

The discordant results between complementary indicators of access in the same population merit discussion. The Institute of Medicine describes access as “...a broad set of concerns that center on the degree to which individuals and groups are able to obtain needed services from the medical system," and "the timely use of personal health services to achieve the best possible health outcomes." Implicit in these definitions is the recognition that access combines the constructs of need for health care, use of appropriate health services, and health outcomes. For all their usefulness, utilization rates, if used alone to gauge equity of access, can be problematic, and may be especially so for populations with high need, such as those with poor health status and complex chronic diseases. ${ }^{9}$ In these circumstances, other utilization indicators, such as coordinated care for chronic conditions, or outcome indicators, such as preventable hospitalization rates for chronic 
conditions, may provide more robust measures of access. In other words, as was apparent in the first two patient anecdotes, simply attending a primary care visit may not be enough to ensure access to appropriate and timely services.

There are additional reasons to suspect that primary care utilization may not provide a comprehensive picture of access for patients with limited HL. Recent work reveals that patients with limited HL report a profound reliance on their primary care physicians (oral communication of unpublished results; Rima Rudd, MD, PhD; February, 2003), and often forego other needed visits or services in favor of the more familiar and established physician, or avoid disturbing or challenging their physician because they perceive the relationship with their physician to be their tenuous link to the larger health care system. Patients with limited HL are less likely to experience visits with their primary care physician as understandable, empowering, or collaborative, ${ }^{10}$ suggesting that the degree of access may not be sufficient to meet patients' health and informational needs. When placing the results of the Baker et al. study ${ }^{1}$ within the context of the other well-done Prudential HL studies, one can conclude that, while Medicare managed care patients with limited HL appear able to navigate the bureaucratic tribulations of connecting with a primary care physician, equal performance on this yardstick of access does not appear to translate into equal performance in anticipated access-related outcomes. Further mining of the Prudential HL Study, and other ongoing HL research, may uncover the reasons for these discrepancies.

John-Baptiste et al. ${ }^{11}$ also make an important contribution to the field with their study of the effect of language barriers on inpatient outcomes of care in 3 hospitals in Toronto, Canada. The authors used an administrative database to analyze 59,547 hospital admissions, each grouped into 1 of 23 different medical and surgical conditions. In multivariate models, LEP patients had significantly longer hospital stays than English-speaking patients for 7 of the 23 conditions, ranging from 0.6 days more for coronary artery bypass surgery to 3.6 days more for stroke. In a meta-analysis of case mix groups, this finding persisted, with LEP patients staying approximately 0.5 days longer than English-speaking patients. While these results have obvious cost implications, does this study indicate that LEP patients experienced lower quality of care? Earlier studies that have explored the impact of language on inpatient care-showing a higher risk of intubation in asthma patients, ${ }^{12,13}$ a lower likelihood of receiving analgesics after cesarean section, ${ }^{14}$ and worse quality of obstetrical care ${ }^{16}$ were limited by a lack of control for possible confounding factors. In this study, John-Baptiste et al. could not determine whether lower quality of care contributed to their findings. The limitations of the database only allowed for exploratory analyzes, such as the degree to which inhospital complications or number of procedures affected the relationship between language status and length of stay, and the extent to which in-hospital mortality differed by language proficiency.
The findings of increased length of stay are consistent with previous studies suggesting that language barriers result in higher utilization of expensive emergency and hospital services. ${ }^{16,17}$ Further examination of factors that mediate prolonged hospitalizations for LEP patients is warranted. Could physicians who do not have access to trained medical interpreters be uncertain or confused about LEP patients' symptoms, leading them to obtain additional studies or continue observation to ensure clinical stability prior to discharge? Could patients be experiencing gaps in communication or errors in interpretation ${ }^{18}$ that lead to worse pain control and anxiety, each of which can prolong hospital stay? ${ }^{19}$ Other issues to consider include difficulties arranging linguistically and culturally appropriate postdischarge care or, as the authors suggest, delays associated with interpretation. Unfortunately, the available administrative data did not allow the authors to ascertain the extent to which patients had access to and/ or used interpreter services. Most hospitals in the United States rely on an amalgam of bilingual providers and a wide variety of interpreters, including telephonic interpreters, bilingual hospital staff (from nurses to custodians), volunteers, patient family members, and friends. ${ }^{20}$ Frequently, there is no interpreter. ${ }^{21}$ Teasing apart the relative impact of different forms of interpretation versus no interpretation is critical to better understanding what interventions may lead to improved care for LEP patients. Ideally, this would entail including in administrative databases standardized measures of both the need for interpreter services (e.g., validated measures of language proficiency for healthcare settings) and the use of these services.

These studies highlight the potential, and some of the current limitations, of health services research to determine the impact of communication barriers on access, utilization, and quality. They reveal impressions of health care systems that have not systematically attended to the communication needs of their more vulnerable patients. Can problems in the daily interactions between patients and providers described by health communication researchers more clearly reveal themselves through the lens of health services research? Health communication research, while providing profound insights into patient-physician communication, has been hampered by a lack of generalizability and an inability to consistently link to important health outcomes. Health services research has been hampered by the challenge of accounting for the inherent complexity of health care delivery. Can we translate the insights from communication researchers into measures that health services researchers can use to systematically capture experiences like those recounted in the patient anecdotes above? We must strive to determine whether such anecdotes represent aberrations or recurrent patterns with even wider implications, if we are to make available the kinds of scientific evidence that can affect practice and policy.-Dean Schillinger, MD, University of California, San Francisco Primary Care Research Center, Open Society Institute Physician Advocacy Fellow, California Literacy, Inc., San Francisco, 
Calif; and Alice Hm Chen, MD, MPH, Staff Physician, Asian Health Services, Open Society Institute Physician Advocacy Fellow, Asian \& Pacific Islander American Health Forum, San Francisco, Calif.

\section{REFERENCES}

1. Baker DW, Gazmararian JA, Williams MV et al. Health literacy and use of outpatient physician services by medicare managed care enrollees. J General Intern Med. 2004;19:215-21.

2. Baker DW, Parker RM, Williams MV, et al. The health care experience of patients with low literacy. Arch Fam Med. 1996;5:329-34.

3. Bennett D, Rothschild R, Schillinger D. Low Literacy High Risk: The Hidden Challenge Facing Health Care in California. Report from the California Health Literacy Initiative; October 2003. Available at: http://www.cahealthliteracy.org. Accessed October 28, 2003.

4. Shuptrine SC, Grant VC. Study of the AFDC/Medicaid eligibility process in the Southern States. Southern Regional Project on Infant Mortality on the Southern Governors' Association and Southern Legislative Conference; April, 1998.

5. Perry M, Kannel S, Valdez RB, Chang C. Medicaid and Children: Overcoming Barriers to Enrollment-Findings from a National Survey. Publication \#2174. Washington, DC: The Kaiser Commission on Medicaid and the Uninsured; January 2000.

6. Baker DW, Parker RM, Williams MV, Clark WS, Nurss J. The relationship of patient reading ability to self-reported health and use of health services. Am J Public Health. 1997;87:1027-30.

7. Scott TL, Gazmararian JA, Williams MV, Baker DW. Health literacy and preventive health care use among medicare enrollees in a managed care organization. Med Care. 2002;40:395-404.

8. Baker DW, Gazmararian JA, Williams MV, et al. Functional health literacy and the risk of hospital admission among medicare managed care enrollees. Am J Public Health. 2002;92:1278-83.

9. Institute of Medicine Committee on Monitoring Access to Personal Health Services. Access to Health Care in America. Washington, DC: National Academy Press; 1993.
10. Schillinger D, Bindman A, Stewart A, Wang F, Piette J. Functional health literacy and the quality of physician-patient communication among diabetes patients. Patient Educ Couns. In Press. Available at: http://dx.doi.org/10.1016/S07383991(03)00107-1. Accessed January, 2004.

11. John-Baptiste A, Naglie G, Tomlinson G, et al. The Effect of English language proficiency on length of stay and in-hospital mortality. J Gen Intern Med. 2004;19:221-38.

12. LeSon $\mathrm{S}$, Gershwin ME. Risk factors for asthmatic patients requiring intubation. I. Observations in children. J Asthma. 1995;32:285-94.

13. LeSon S, Eric GM. Risk factors for asthmatic patients requiring intubation. II. Observations in teenagers. J Asthma. 1995;32:379-89.

14. Carnie JC, Perks D. The pattern of postoperative analgesic administration in non-English speaking Asian women following caesarian section. Ann R Coll Surg Engl. 1984;66:365-6.

15. Devore JS, Koskela K. The language barrier in obstetric anesthesia. Am J Obstet Gynecol. 1980;137:745-6.

16. Hampers LC, Cha S, Gutglass DJ, Binns HJ, Krug SE. Language barriers and resource utilization in a pediatric emergency department. Pediatrics. 1999;103:1253-6.

17. Lee ED, Rosenberg CR, Sixsmith DM, Pang D, Abularrage J. Does a physician-patient language difference increase the probability of hospital admission? Acad Emerg Med. 1998;5:86-9.

18. Flores G, Laws MB, Mayo SJ, et al. Errors in medical interpretation and their potential consequences in pediatric encounters. Pediatrics. 2003;111:6-14.

19. Egbert LD, Battit GE, Welch CE, Bartlett MK. Reduction of postoperative pain by encouragement and instruction of patients. A study of doctor-patient rapport. N Engl J Med. 1964;270:825-27.

20. Ginsberg C, Martin V, Andrulis D, Shaw-Taylor Y, McGregor C. Interpretation and Translation Services in Health Care: A Survey of US Public and Private Teaching Hospitals. Washington, DC: National Public Health and Hospital Institute; 1995.

21. Andrulis D, Goodman N, Pryor C. What a Difference an Interpreter Can Make. Health Care Experiences of Uninsured with Limited English Proficiency. The Access Project. Waltham, Mass: Brandeis University; 2002. 